\title{
Numerical Modeling of the Flash Boiling Characteristics of E-Fuels at Low Ambient Pressure
}

\author{
Avijit Saha*1, Abhishek. Y. Deshmukh ${ }^{1}$, Temistocle Grenga ${ }^{1}$, Mathis Bode ${ }^{1}$, Mathias \\ Grunewald $^{2}$, Yakup Kaya ${ }^{2}$, Valeri Kirsch ${ }^{2}$, Manuel A. Reddemann ${ }^{2}$, Reinhold Kneer ${ }^{2}$, Heinz \\ Pitsch ${ }^{1}$ \\ ${ }^{1}$ Institute for Combustion Technology, RWTH Aachen University, Aachen, Germany \\ ${ }^{2}$ Institute of Heat and Mass Transfer, RWTH Aachen University, Aachen, Germany \\ ${ }^{*}$ Corresponding author email: a.saha@itv.rwth-aachen.de
}

\begin{abstract}
E-fuels based on renewable electricity and carbon sources from $\mathrm{CO}_{2}$ have gained much attention in recent years due to ever-increasing stringent emission regulations. Some of these e-fuels have high vapor pressure, which makes them more prone to flash boiling for some operating conditions. In this work, the flash boiling phenomenon of these novel e-fuels is first investigated at the single droplet level using dimethyl ether (DME) as a generic example. A Lagrangian Particle Tracking approach is employed to simulate the combined internal and external flash vaporization of a DME single droplet. The simulation results reveal that internal vaporization via bubble nucleation and growth is the primary source causing the transition of the metastable liquid phase to the stable one. An analysis of the non-dimensional Rayleigh-Plesset equation is performed to estimate the relative importance of different forces in determining the bubble growth dynamics of a flash boiling droplet. Based on the analysis, approximate analytical solutions of the Rayleigh-Plesset equation are derived.
\end{abstract}

\section{Keywords}

E-fuels, Flash boiling, Lagrangian particle tracking, Bubble dynamics

\section{Introduction}

According to the Intergovernmental Panel on Climate Change (IPCC) report, greenhouse gas (GHG) emissions (especially $\mathrm{CO}_{2}$ ) from the transportation sector are expected to increase at a faster rate than any other energy end-use sectors unless sufficient measures are undertaken to develop highly efficient and clean combustion systems [1]. In this context, concerted efforts are being made to develop e-fuels from renewable electricity and $\mathrm{CO}_{2}$ as carbon sources. The thermo-physical properties of these fuels are exceedingly different from conventional fuels. For example, dimethyl ether (DME) is one such e-fuel candidate, which has emerged as a promising substitute to conventional fuels as it produces almost no soot during combustion [2]. However, the higher vapor pressure of DME compared to conventional fuels makes it more susceptible to flash boiling while operating in an environment where the saturation pressure of the liquid fuel is higher than the surrounding gas pressure. Although flash boiling is known as one of the most effective means to obtain high-quality liquid atomization, it may lead to an adverse impact on the spray characteristics depending on the degree of superheating and spray collapse [3]. Flash boiling is a fairly complex phenomenon involving several sub-processes such as bubble nucleation, growth, and droplet burst. These processes occur on a time-scale of $1-100 \mu \mathrm{s}$ [3]. Thus, it is difficult to accurately quantify the characteristics of flash boiling fuel sprays unless the physics underlying the flash boiling process are explored in detail at the microscopic single droplet level. 
Flash boiling liquid droplets not only undergo phase transition internally via bubble formation but also from its external surface. In the past, many efforts with varying degrees of simplifying assumptions have been made to explore the dynamics of a single vapor bubble in an infinite pool of superheated liquid. Rayleigh [4] studied the inertia-dominated growth of a spherical vapor bubble, neglecting the cooling effect caused by vaporization at the bubble surface. Plesset and Zwick [5] proposed an asymptotic solution for the thermal diffusion-controlled bubble growth, which is only valid at the later stages of growth. Mikic et al. [6] combined these two regimes to formulate a general expression for bubble growth. Their simulation results showed good agreement with the experimental measurements over a wide range of system pressures. Robinson [7] later studied the effect of interaction between hydrodynamics and thermal diffusion near the liquid-vapor interface on different phases of bubble growth. However, single droplet flash boiling combining internal and external flash vaporization has not been much studied earlier. Recently, Yang [8] studied single droplet as well as spray flash boiling phenomena combining both sources of vaporization. Nevertheless, the fundamental physics of the bubble dynamics were not explored. Xi et al. [9] investigated the bubble dynamics of a flash boiling DME single droplet neglecting the external flash vaporization and conduction thermal resistance in the droplet.

Therefore, in this work, a flash boiling model combining internal and external flash vaporization processes is implemented in a Lagrangian Particle Tracking (LPT) framework to study the single droplet flash boiling of DME. An analysis of the non-dimensional Rayleigh-Plesset (RP) equation is performed to identify the relative influence of hydrodynamic, surface tension, and viscous forces in determining the growth characteristics of vapor bubbles. Based on the analysis, approximate analytical solutions of the RP equation are derived. Simulation results are discussed in two parts. First, the detailed bubble growth dynamics of DME droplet is presented, followed by the relative importance of internal flashing on overall droplet vaporization. Second, the non-dimensional analysis and analytical solutions are discussed.

\section{Numerical methods}

A three-dimensional two-way coupled Eulerian-Lagrangian numerical framework is used in this study. An in-house code CIAO, which is a structured, high-order, finite-difference code, is used to solve the compressible Navier-Stokes equations. In this section, the equations governing the Lagrangian particle phase will be described briefly. The reader is referred to Saha et al. [12] for more details about the flash boiling models in CIAO's LPT solver. Detailed description of numerical methods in CIAO can be found in Desjardins et al. [10] and Mittal et al. [20].

Droplet vaporization models: A flash boiling single droplet undergoes spontaneous phase transition via the formation of vapor bubble nuclei. The newly formed vapor bubble nuclei, whose radii are smaller than the critical value, continue to grow until a void fraction value of 0.55 is reached [13]. The growth process of a spherically symmetric vapor bubble can be described through the RP equation as [14]

$$
\underbrace{P_{v}\left(T_{v}\right)-P_{\infty}}_{\Delta P}=\underbrace{\rho_{l} R \frac{d^{2} R}{d t^{2}}+\frac{3}{2} \rho_{l}\left(\frac{d R}{d t}\right)^{2}}_{P_{h d}}+\underbrace{\frac{4 \mu_{l}}{R} \frac{d R}{d t}}_{P_{\mu}}+\underbrace{\frac{2 \sigma}{R}}_{P_{\sigma}},
$$

where $\rho_{l}$ is the liquid density, $R$ the bubble radius, $\sigma$ the surface tension of the liquid, $\mu_{l}$ the viscosity of the liquid, $P_{v}$ the saturated vapor pressure at vapor temperature $T_{v}$, and $P_{\infty}$ the liquid pressure, which is assumed equal to the surrounding gas pressure, $P_{g}$, neglecting the 
surface tension between droplet and gas. In this study, $T_{v}$ is assumed to be equal to the liquid temperature at the bubble surface. In Equation 1, $\Delta P$ denotes the pressure difference, $P_{h d}$ represents the hydrodynamic pressure resulting from the bulk liquid motion, $P_{\sigma}$ indicates the pressure due to the surface tension force, and $P_{\mu}$ represents the viscous force at the liquidvapor interface.

A thin thermal boundary-layer assumption is employed to model the $T_{v}$ as [15]

$$
T_{v}=T_{d}-2\left(\frac{\pi t}{12 \alpha}\right)^{1 / 2} \frac{L_{v} \rho_{v}}{\rho_{l} c_{l}} \frac{d R}{d t},
$$

where $T_{d}$ is the droplet's bulk temperature, $\alpha$ the thermal diffusivity of the liquid, $\rho_{v}$ the saturated vapor density, $L_{v}$ the latent heat of vaporization at $T_{v}$, and $c_{l}$ the liquid specific heat capacity. $t$ in Equation 2 denotes time, which is measured from the point of inception of bubble growth.

Phase transition may also occur from the external surface of a flash boiling droplet due to the heat transfer from the droplet interior and from the surrounding gas. The vaporization rate due to the internal heat transfer is modeled as [16]

$$
\dot{m}_{f}=\frac{h \Delta \theta A}{L_{v}\left(T_{b}\right)},
$$

where $\dot{m}_{f}$ represents the flash-boiled vapor mass flow rate, $h$ the heat transfer coefficient, $A$ the droplet surface area, and $L_{v}$ the latent heat of vaporization at droplet boiling temperature, $T_{b} . \Delta \theta$ denotes the degree of superheating, $\Delta \theta=\left(T_{d}-T_{b}\right)$. In this study, $h$ is estimated using the following empirical functions proposed by Adachi et al. [16],

$$
h= \begin{cases}0.76\left(T_{\mathrm{d}}-T_{\mathrm{b}}\right)^{0.26} & \left(0<T_{\mathrm{d}}-T_{\mathrm{b}}<5\right) \\ 0.027\left(T_{\mathrm{d}}-T_{\mathrm{b}}\right)^{2.33} & \left(5<T_{\mathrm{d}}-T_{\mathrm{b}}<25\right) \\ 13.8\left(T_{\mathrm{d}}-T_{\mathrm{b}}\right)^{0.39} & \left(T_{\mathrm{d}}-T_{\mathrm{b}}>25\right)\end{cases}
$$

The mass flow rate due to the external heat transfer is modeled as [17]

$$
\dot{m}_{e x}=2 \pi \frac{\lambda_{g}}{c_{p}} r_{d} \frac{N u}{\left(1+\frac{\dot{m}_{f}}{\dot{m}_{e x}}\right)} \ln \left[1+\left(1+\frac{\dot{m}_{f}}{\dot{m}_{e x}}\right) \frac{c_{p}\left(T_{g}-T_{s}\right)}{L_{v}\left(T_{s}\right)}\right] .
$$

$\lambda_{g}$ and $c_{p}$ denote the gas-phase thermal conductivity and specific heat capacity at constant pressure, respectively, obtained using one-third rule. $N u$ is the Nusselt number, $T_{s}$ the droplet surface temperature, which remains equal to $T_{b}$ until the superheated liquid reaches its equilibrium state, $T_{g}$ the gas-phase temperature, and $r_{d}$ the droplet radius.

The infinite conductivity model is used to obtain the droplet bulk temperature as

$$
\frac{d T_{d}}{d t}=\frac{N u}{3 \operatorname{Pr}} \frac{c_{p}}{c_{l}} \frac{f_{2}}{\tau_{d}}\left(T_{g}-T_{s}\right)-\frac{\dot{m}_{t} L_{v}\left(T_{s}\right)}{m_{d} c_{l}},
$$

where $m_{\mathrm{d}}$ is the droplet mass, $\operatorname{Pr}$ the Prandtl number, and $\tau_{\mathrm{d}}$ the particle time constant defined as $\tau_{\mathrm{d}}=\rho_{\mathrm{l}} d_{\mathrm{d}}^{2} /\left(18 \mu_{\mathrm{g}}\right)$, where $d_{\mathrm{d}}$ is the diameter of the droplet and $\mu_{\mathrm{g}}$ the dynamic viscosity of the surrounding gas. The factor $f_{2}$ denotes an analytical evaporative heat transfer correction to the solid sphere Nusselt number [18]:

$$
f_{2}=\beta /(\exp (\beta)-1), \quad \beta=-\left(\frac{3 \operatorname{Pr}_{\mathrm{g}} \tau_{\mathrm{d}}}{2}\right) \frac{\dot{m}_{\mathrm{t}}}{m_{\mathrm{d}}} .
$$


Non-dimensionalization of Rayleigh-Plesset equation: The Rayleigh-Plesset equation is nondimensionalized using the following scales:

$$
R^{*}=\frac{R}{R_{c}} ; t^{*}=\frac{v_{R L}^{2} t}{R_{c r i t}} ; \rho^{*}=\frac{\rho_{l}}{\rho_{l 0}} ; P^{*}=\frac{p}{\rho_{l 0} v_{R L}^{2}} ; \sigma^{*}=\frac{\sigma}{\sigma_{l 0}} ; \mu^{*}=\frac{\mu_{l}}{\mu_{l 0}}
$$

where ' $*$ ' denotes the non-dimensional quantities and subscript ' 0 ' the initial values. $R_{c}$ is the critical radius given by $R_{c}=2 \sigma_{l 0} /\left(P_{v 0}-P_{g 0}\right)$ and $v_{R L}$ the Rayleigh solution for bubble growth rate, $v_{R L}=\sqrt{(2 / 3)\left(P_{v 0}-P_{g 0}\right) / \rho_{l 0}}$. Substituting the above relations into Equation 1 yields the non-dimensional Rayleigh-Plesset equation:

$$
P_{v}^{*}\left(T_{v}\right)-P_{g}^{*}=\rho_{l}^{*} R^{*} \frac{d^{2} R^{*}}{d t^{* 2}}+\frac{3}{2} \rho_{l}^{*}\left(\frac{d R^{*}}{d t^{*}}\right)^{2}+\frac{4 \mu_{l}^{*}}{R^{*} R e} \frac{d R^{*}}{d t^{*}}+\frac{2 \sigma^{*}}{R^{*} W e},
$$

Simplification of Equation 8 by neglecting the external flash vaporization $\left(T_{d}=T_{d 0}\right)$ and cooling effect of vaporization $\left(T_{v}=T_{d 0}, \mu^{*}, \sigma^{*}\right.$, and $\left.\rho_{l}^{*}=1\right)$ yields

$$
\underbrace{P_{v}^{*}\left(T_{d 0}\right)-P_{g}^{*}}_{\Delta P^{*}}=\underbrace{R^{*} \frac{d^{2} R^{*}}{d t^{* 2}}}_{P_{h d, a c c}^{*}}+\underbrace{\frac{3}{2}\left(\frac{d R^{*}}{d t^{*}}\right)^{2}}_{P_{h d, v e l}^{*}}+\underbrace{\frac{4}{R^{*} R e} \frac{d R^{*}}{d t^{*}}}_{P_{\mu}^{*}}+\underbrace{\frac{2}{R^{*} W e}}_{P_{\sigma}^{*}},
$$

$R e$ is Reynolds number, $R e=\rho_{l 0} v_{R L} R_{c} / \mu_{l 0}$ and $W e$ the Weber number, $W e=\rho_{l 0} v_{R L}^{2} R_{c} / \sigma_{l 0}=$ 1.34 .

\section{Results and discussion}

Single droplet case: Figure 1 shows the bubble growth characteristics of a flash boiling DME droplet at $\Delta \theta=27.85 \mathrm{~K}$. The droplet size is similar to the nozzle exit diameter. Three distinct growth phases are observed: (1) surface tension-controlled, (2) transition, and (3) inertiacontrolled growth phase.
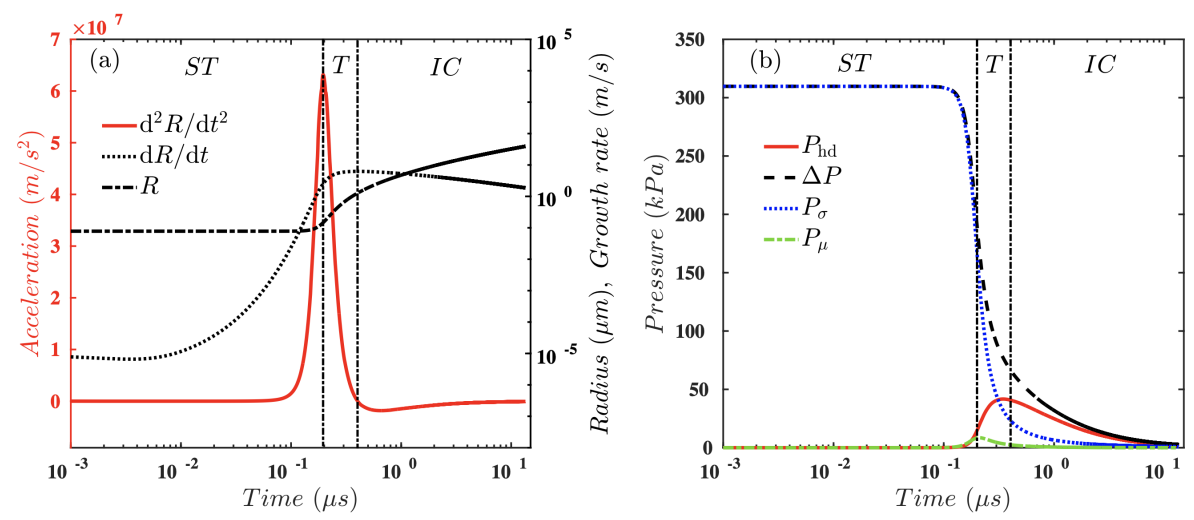

Figure 1. Simulated bubble growth characteristics of DME single droplet for $P_{g}=2$ bar, $T_{g}=293 \mathrm{~K}$, and $\Delta \theta=$ $27.85 \mathrm{~K}$. Subfigure (a) illustrates the bubble radius, growth rate, and interface acceleration over time. Subfigure (b) depicts the variation of different pressure force terms in Rayleigh-Plesset equation.

Surface tension acts as a rate-limiting factor determining the bubble growth at the very beginning. This is because the vapor bubbles, which have just nucleated, are only $10^{-7} \%$ larger than the $R_{c}$ leading to a large value of $P_{\sigma}$ such that it is almost balanced by $\Delta P$, as shown in Figure $1 \mathrm{~b}$. The change in bubble radius is very small at the beginning of the surface tensioncontrolled growth phase. However, rapid interface acceleration with increasingly larger radius is observed in the latter period of this growth phase (Figure 1a), which in turn slowly generates the liquid motion, as shown from increased $P_{h d}$ in Figure 1b. The time at which maximum 
interface acceleration occurs marks the end of the surface tension-controlled growth and the onset of the transition phase. The transition growth phase is characterized by a sharp decrease in $P_{v}$ together with an increasing $P_{h d}$ (Figure 1b). The bubble radius undergoes a significant increase in this growth phase, which is also the reason for the sharp decrease in $P_{\sigma}$. At the end of the transition phase, $P_{\sigma}$ becomes insignificant and $P_{h d}$ increases to such an extent that $\Delta P \approx P_{h d}$. The peak value of $P_{h d}$ also occurs in the later period of the transition phase depicting moderate liquid motion surrounding the liquid-vapor interface. The transition phase ends at $t=0.40 \mu \mathrm{s}$, where the bubble growth reaches its maximum value. After this phase, inertia-controlled growth begins, where the ability of the expanding interface to push back the surrounding liquid is considered to be a rate-limiting factor. The pressure differential, $\Delta P$, remains balanced by the hydrodynamic pressure, $P_{h d}$, throughout the inertia-controlled growth phase. The pressure force due to the viscosity of the liquid, $P_{\mu}$, remains negligible during the bubble expansion.

The mass flow rate of DME fuel vapor is shown in Figure 2 for $P_{g}=2$ bar, $T_{g}=293 \mathrm{~K}$, and $\Delta \theta=$ $27.85 \mathrm{~K}$. Figure 2 a depicts that during the initial stage of bubble growth, $\dot{m}_{i}$ remains smaller than $\dot{m}_{t}$ due to extremely small vapor bubble nuclei. As the bubbles gradually expand, $\dot{m}_{i}$ increases and starts dominating over $\dot{m}_{t}$ near the end of the transition growth phase, illustrating $\dot{m}_{i}$ is the primary source of vaporization in the superheated regime. Figure $2 \mathrm{~b}$ illustrates the relative contribution of internal as well as external-heat-transfer-driven vapor mass flow in the total mass leaving the external droplet surface $\left(\dot{m}_{t}\right)$. It is observed that $\dot{m}_{f}$ increases with time. This is because of the increase in droplet surface area due to the expansion of the vapor bubbles, as depicted in Figure 3 . The increasing $\dot{m}_{f}$ will in turn reduce the heat flux from the surrounding gas resulting in a decreasing trend in $\dot{m}_{e x}$ (as shown in Figure $2 b$ ).
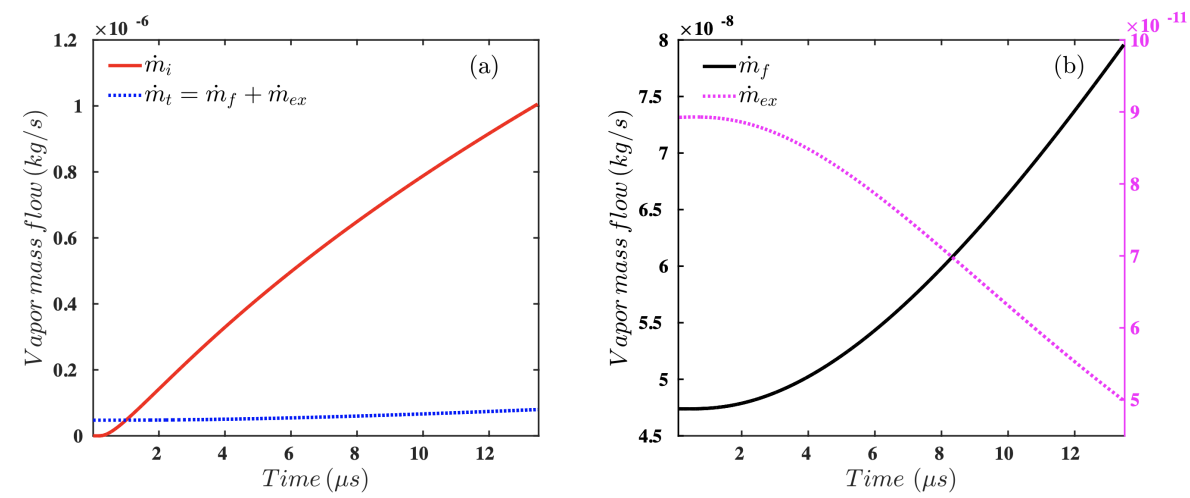

Figure 2. Fuel vapor mass flow rate of DME single droplet: (a) Variation of internally $\left(\dot{m}_{i}\right)$ and externally $\left(\dot{m}_{t}\right)$ vaporized fuel vapor mass flow, (b) Relative contribution of $\dot{m}_{f}$ and $\dot{m}_{\text {ex }}$ in overall external surface vaporization.

Non-dimensional analysis of bubble dynamics: Figure 4 illustrates the effect of varying Reynolds number on different non-dimensional pressure force terms in Equation 9. The increase of $R e$ leads to a dramatic reduction in $P_{\mu}^{*}$, as shown in Figure 4b. The maximum value of $P_{\mu}^{*}$ decreases about $98 \%$ as the $R e$ is increased from 1 to 100 . The low viscous frictional resistance in turn, shortens the growth delay time, which is defined as time interval between the initial perturbation and when the bubble radius undergoes a noticeable increase. Due to shorter delay time, a sharp decline in $P_{\sigma}^{*}$ occurs relatively quickly with increasing $R e$. A notable increase in $P_{h d, a c c}^{*}$ and $P_{h d, v e l}^{*}$ is also observed with increasing $R e$. The peak value of $P_{h d, a c c}^{*}$ is increased by about $65 \%$ compared to the value at $R e=1$. 


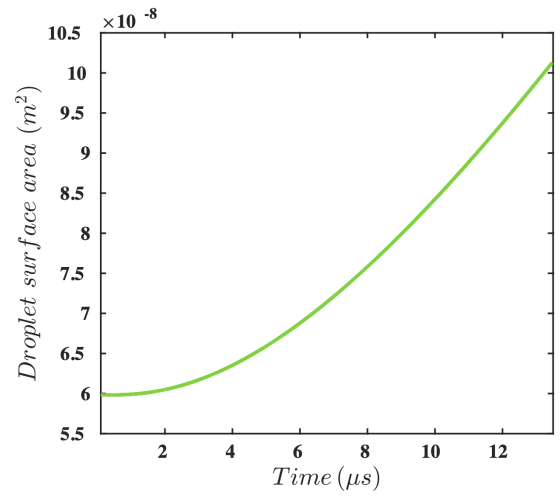

Figure 3. Variation of superheated DME droplet surface area.

$$
-R e=1 \cdots \cdot R e=10--\cdot R e=100
$$
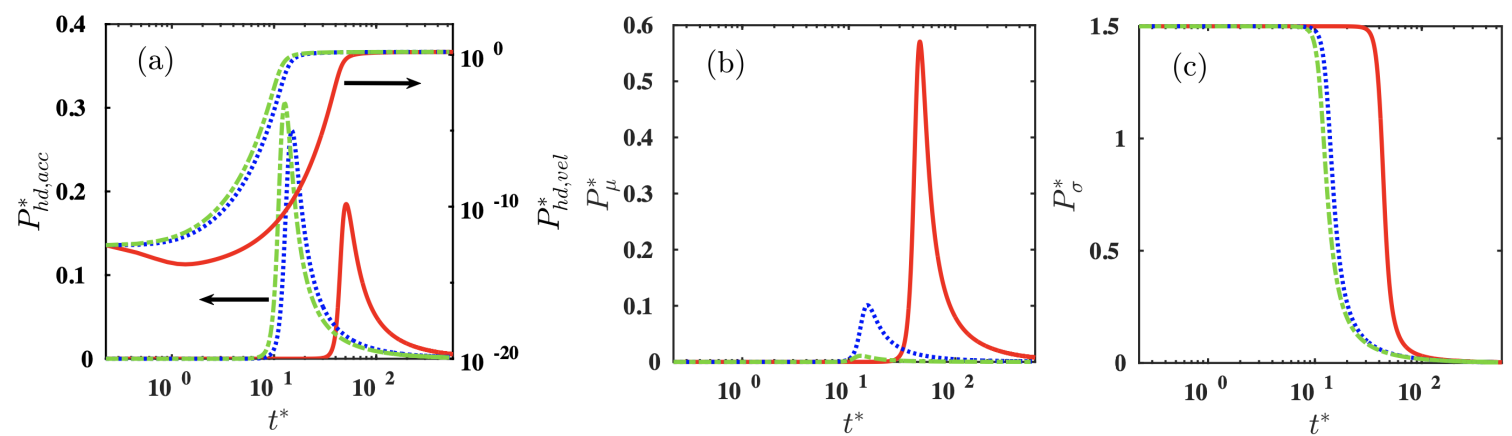

Figure 4. Influence of $R e$ on bubble growth characteristics. Subfigure (a) shows the variation of non-dimensional inertia force due to acceleration $\left(P_{h d, a c c}^{*}\right)$ and velocity $\left(P_{h d, v e l}^{*}\right)$. Subfigures (b) and (c) illustrate the variation of non-dimensional viscous force and surface tension force, respectively.

Approximate analytical solutions of RP equation: The RP equation is generally solved numerically due to the difficulty associated with obtaining the analytical solutions. However, the slope of $\dot{R}$ may become very steep in some portion of the growth curve depending on the superheating degree of the system [12], which requires extremely small time steps $\left(<10^{-11} s\right)$ to prevent unrealistic $T_{v}$ at the liquid-vapor interface. Alehossein and Qin [19] proposed a variable time step method to accurately capture such a steep gradient in the growth curve. Alternatively, it is possible to construct an approximate analytical solution of the RP equation. However, the accuracy of the predicted bubble size will highly depend on the degree of simplifying assumptions. For example, the non-dimensional analysis presented above depicts that the effect of viscous force on the bubble dynamics becomes negligible with increasing $R e$. Thus, $\dot{R}^{*}$ corresponding to high $R e$ can be expressed as [14]

$$
\dot{R}^{*}=\sqrt{\frac{2}{3} \Delta P^{*}\left\{1-\left(\frac{1}{R^{*}}\right)^{3}\right\}-\frac{2}{R^{*} W e}\left\{1-\left(\frac{1}{R^{*}}\right)^{2}\right\} .}
$$

Conversely, at low $R e$, the effect of $P_{h d, a c c}^{*}$ becomes small, thus neglecting this term yields the following expression of $\dot{R}^{*}$ for low $R e$,

$$
\dot{R}^{*}=-\frac{4}{3 R^{*} R e}+\sqrt{\left(\frac{4}{3 R^{*} R e}\right)^{2}-\left\{\frac{4}{3 R^{*} W e}-\frac{2}{3} \Delta P^{*}\right\}} .
$$


A comparison of the numerical and approximate analytical solutions is shown in Figure 5 for $R e=1,10$, and 100. It is observed that the bubble radius predicted using Equation 10 becomes closer to the numerical solution as $R e$ is increased to the higher values. In contrast, Equation 11 predicts the bubble radius more accurately as $R e$ is decreased to 1 .

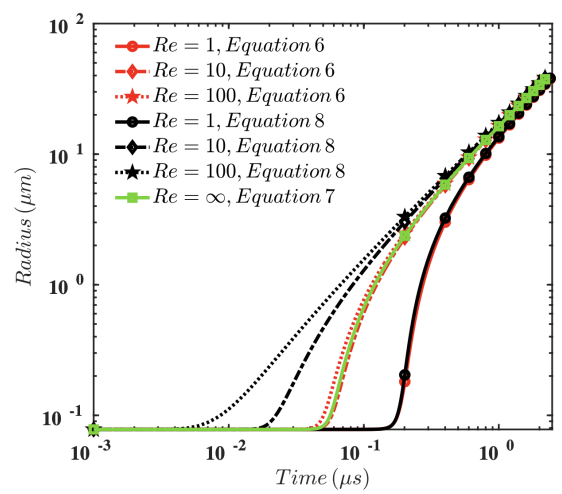

Figure 5. Comparison of numerically predicted bubble radius with the analytical solution.

\section{Conclusions}

In this study, first, flash boiling of a DME single droplet combining internal and external flash vaporization processes was investigated. Simulation results showed three distinct growth phases of the vapor bubbles: (1) surface tension-controlled, (2) transition, and (3) inertia-controlled growth phase. It was found that the internal vaporization via formation and subsequent growth of the bubbles is the primary source causing the metastable liquid to attain its equilibrium state. Second, a non-dimensional analysis of the RP equation was performed to discover the relative importance of the terms representing the different physical phenomena associated with the bubble dynamics of a flash boiling droplet for low and high Re. Based on this analysis, different analytical solutions of the RP equation were proposed for the high and low Re regimes, respectively. Bubble radius obtained using analytical solution was shown to be in good agreement with the numerical solution.

\section{Acknowledgements}

The numerical simulations were performed as part of the Cluster of Excellence "The Fuel Science Center", which is funded by the Deutsche Forschungsgemeinschaft (DFG, German Research Foundation) under Germany's Excellence Strategy - Exzellenzcluster 2186 "The Fuel Science Center" ID: 390919832.

\section{References}

[1] Intergovernmental Panel on Climate Change. (2015). Transport. In Climate Change 2014: Mitigation of Climate Change: Working Group III Contribution to the IPCC Fifth Assessment Report,pp. 599-670, Cambridge: Cambridge University Press.

[2] Kim, M. Y., Yoon, S. H., Ryu, B. W, Lee, C. S, 2008, Fuel, 87, pp. 2779-2786.

[3] Price, C., Hamzehloo, A., Aleiferis, P., Richardson, D., 2018, Fuel, 221, pp. 518-541.

[4] Strutt, J. W., 2009, Vol. 6, pp. 504-507, Cambridge Library Collection - Mathematics, Cambridge University Press. 
[5] Plesset, M. S., Zwick, A., 1954, J. Appl. Phys., 25(4), pp. 493-500.

[6] Mikic, B. B., Rohsenow, W. M., Griffith, P., 1970, Int. J. Heat Mass Transf., 13, pp. 657-666.

[7] Robinson, A. J., 2002, "Bubble Growth Dynamics in Boiling". PhD Thesis.

[8] Yang, S., 2017, Atomization and Sprays, 27 (11), pp. 963-997.

[9] Xi, X., Liu, H., Jia, M., Xie, M., Yin, H., 2017, Int. J. Heat Mass Transf., 107, pp. 1129-1137.

[10] Desjardins, O., Blanquart, G., Balarac, G., Pitsch, H., 2008, J. Comput. Phys., 227 (15), pp.7125-7169.

[11] Bode, M., Kaya, Y., Reddemann, M. A., Goeb, D., Kirsch, V., Kneer, R., Pitsch, H., Reuter, U., Bebber, D., Herudek, C., Sommerhoff, A., Willems, W., 2018,THIESEL 2018 Conference on Thermo- and Fluid Dynamic Processes in Direct Injection Engines.

[12] Saha, A., Grenga, T., Deshmukh, A. Y., Hinrichs, J., Bode, M., Pitsch, H., 2021, Submitted to Fuel.

[13] Kawano, D., Goto, Y., Odaka, M., Senda, J., 2004,SAE Tech. Papers.

[14] Brennen, C. E., 2013, Cavitation and bubble dynamics, Cambridge University Press.

[15] Board, S. J., Duffey, R. B., 1971, Chemical Engineering Science, 26(3), pp. 263-274.

[16] Adachi, M., McDonell, V. G., Tanaka, D., Senda, J., Fujimoto, H., 1997, SAE Tech. Papers.

[17] Zuo, B., Gomes, A. M., Rutland, C. J., 2000, Int. J. Engine Res., 1 (4), pp.321-336.

[18] Miller, R. S., Bellan, J.,1999, J. Fluid Mech., 384, pp.293-338.

[19] Alehossein, H., Qin, Z., 2007, Int. J. Numer. Meth. Eng., 72, pp. 780-807.

[20] Mittal, V., Kang, S., Dorna, E., Cook, D., Pitsch, H., 2014, Oil \& Gas Science and Technology - Revue d'IFP Energies nouvelles, 69 (1), pp. 29-40. 